\section{Computertools im Prozeß von ECODESIGN}

\author{
von Robert Wimmer, GrAT, TU Wien
}

Bewertungsverfahren und unterstützende Instrumente zu ihrer Operationalisierung sind ein zentrales Anliegen, um das die Diskussion um umweltgerechte Produktgestaltung und praktikable Handlungs- und Eingriffsmöglichkeiten immer wieder kreist.

Jeder/e kennt Beispiele aus der eigenen Erfahrung oder der öffentlichen Diskussion, die sich dadurch auszeichnen, recht widersprüchliche Resultate zu produzieren. Sei es der Streit um das "umweltfreundlichere Produkt oder Material", Kunststoff oder Papier, Ein- oder Mehrwegverpackung, oder die Quantifizierung der erforderlichen Ressourcenreduktion für eine nachhaltige Entwicklung (Faktor 10, 4, 2 oder ?).

Von immer mehr Firmen wird jedoch die (auch ökonomische) Chance erkannt, die vorsorgende Umweltschutzmaßnahmen durch ihr großes Potential an Ressourceneinsparung bergen, und es besteht ein zunehmender Bedarf an systematischer
Unterstützung in den damit verbundenen Planungs- und Bewertungsaufgaben.

Parallel dazu gibt es ein vielfältiges, aber wenig strukturiertes Angebot von Hilfsmitteln mit unterschiedlichen Möglichkeiten und Zielsetzungen.

Die Zahl der Computerprogramme im Bereich betrieblicher Umweltmaßnahmen ist seit Beginn der 90er Jahre sprunghaft angestiegen.

Aus diesen Gründen wurde im Rahmen des Forschungsschwerpunkts ECODESIGN der Gruppe Angepaßte Technologie (GrAT) an der TU Wien im Auftrag des österreichischen Ministeriums für Umwelt, Jugend und Familie eine umfangreiche Untersuchung von Computerprogrammen für präventiven Umweltschutz durchgeführt: "Software-Recherche und Test von Programmen zum betrieblichen Einsatz, für die Bewertung und Umsetzung vorsorgender Umweltschutzmaßnahmen" (ECODESIGN Software Studie).

\section{Zielsetzung und Methodik}

Der gewählte Standpunkt für die Untersuchung war die Anwendbarkeit der angebotenen Hilfsmittel für den Prozeß ECODESIGN. Im Zentrum stand dabei die Frage: Welche Bereiche vorsorgender Umweltschutzmaßnahmen können vom Programm unterstützt werden, und welche Methodik wird dabei verwendet?

Die Ergebnisse sollen einerseits für potentielle Softwareanwender als Überblick und Auswahlhilfe dienen, andererseits einen Input liefern für die methodische Weiterentwicklung der unterstützenden Computertools, etwa durch das Aufzeigen von Defiziten bzw. blinden Flecken.

Dies erforderte einen breiteren Zugang als ein Test von Programmen für einen speziellen, genau definierten, Anwendungsfall.

Es wurde daher in der Auswertung bewußt auf die Ermittlung eines Testsiegers verzichtet, und auf eine transparente und nachvollziehbare Testdurchführung und Dokumentation wert gelegt.

\section{Programmrecherche}

In der international durchgeführten Recherche wird ein umfassender Überblick geboten über alle (Stand vom Juni 1997) am Markt erhältli- 
chen, bzw. in Entwicklung befindlichen Programme. Sie umfaßt eine Liste von ca. 130 erhältlichen Programmen und Kontaktadressen.

\section{Programmbeschreibungen}

Für die näher untersuchten Programme (10 Voll- und 5 Demoversionen) erfolgte eine qualitative Beschreibung der Programme unter Berücksichtigung der folgenden Punkte:

- Kurzbeschreibung der Methodik und des Programmes

- Tabellarischer Steckbrief wichtiger Informationen wie Preise und angebotene Lizenzen, Informationen über die weiterführenden Betreuungsmöglichkeiten und zusätzlichen Dienste, Anwenderempfehlung, Adressen, Demoprogramme.

\section{Programmtests}

In Form von Kriterientests wurde erhoben, welche Lebenszyklusbereiche erfaßbar sind, welche Möglichkeiten die gewählte Datenverarbeitung bietet, ob die Handhabung und Bedienung mit vertretbarem Aufwand erfolgen kann, die Ergebnisse nachvollziehbar und transparent sind, und ob die Quelle für die verwendeten Daten klar erkennbar ist.

Es wurden sowohl allgemeine Softwarekriterien als auch inhaltliche Kriterien berücksichtigt.

Die insgesamt 96 Testkriterien sind in die folgenden acht Testkategorien gegliedert:

- Benutzerhandbuch

- Allgemeine Softwarekriterien

- Flexibilität

- Transparenz

- Berücksichtigte Lebenszyklus-Bereiche und berücksichtigte Faktoren

- Möglichkeiten der Methodik und Ergebnisdarstellung

- Datenumfang und -qualität

- Bedienungsaufwand und Synergien.

Jede dieser Kategorien wurde mit einem Satz von Testfragen auf das jeweilige Programm angewandt, und eine relative Bewertung zwischen 0 und 10 vergeben. Das Ergebnis wurde jeweils begründet und in einer Feedbackrunde wurden von den Softwareanbietern Kommentare zu den Ergebnissen eingeholt.

In teils intensiven Diskussionen wurden die Standpunkte abgeglichen, und um unterschiedliche Standpunkte auch dem Leser der Studie zugänglich $\mathrm{zu}$ machen, wurden die Kommentare der Software Anbieter in den Testbericht mit aufgenommen.

Für eine übersichtliche Darstellung der Ergebnisse des Kriterientests wurde eine semiquantitative Auswertung in Form von Netzdiagrammen durchgeführt.

$\mathrm{Da}$ die Anforderungen potentieller Anwender an das Programm sehr unterschiedlich sein können, stellt dies eine Möglichkeit dar, das Programm hinsichtlich des eigenen Anforderungsprofils zu beurteilen.

\section{Anwendungsbeispiel}

Zusätzlich zu den Kriterientests wurde versucht, anhand eines Beispiels die verschiedenen $\mathrm{Zu}$ gänge der Programmhandhabung zu illustrieren.

Das ausgewählte Beispielprodukt, eine Küchenmaschine, wurde mit einem relativ einfach zu recherchierenden Satz von Daten, wie er von jeder interessierten Firma mit vertretbarem Aufwand bereitgestellt werden kann, beschrieben und die Programmanbieter gebeten, anhand dieser Angaben eine Kalkulation der "Umweltverträglichkeit" durchzuführen.

Die Auswertungen der Ergebnisse variieren zwischen aggregierten Übersichtswerten, Vergleichen mit fiktiven Konkurrenzprodukten und Sensitivitätsanalysen in bezug auf einzelne Umweltauswirkungen.

Generell wurde betont, daß eine möglichst präzise Fragestellung und eine detaillierte Datenaufbereitung die Aussagekraft der Berechnungsergebnisse wesentlich verbessert.

\section{Einsatz von Softwareprogrammen im Rah- men von ECODESIGN}

Aus der Sicht von ECODESIGN werden vor allem die Bereiche Produktionsoptimierung und Produktoptimierung unterstützt.

Dabei sind für die Abbildung komplexer Produktionsabläufe Stofffluß-Modellierungsprogramme am besten geeignet. Für einen Vergleich von Produkten und Sensitivitätsanalysen hinsichtlich einzelner Umweltauswirkungen 
empfiehlt sich die Anwendung eines LCAProgramms. Ansatzweise ist es über die Definition einer funktionellen Einheit auch möglich, Variantenvergleiche auf den Nutzen der untersuchten Produkte zu beziehen.

Die Möglichkeit, den Nutzen der Produkte, die "Produkt-Dienstleistung", ins Zentrum der Betrachtung zu rücken, wie dies etwa für einen Vergleich von Produkten im Verkauf mit Servicekonzepten wünschenswert wäre, steht allerdings noch am Anfang.

Für den Einsatz von Softwareprogrammen im Rahmen von ECODESIGN lassen sich folgende Punkte zusammenfassen:

- Die Ergebnisse von computergestützten Lebenszyklusanalysen (LCA) und Stoffflußmodellierungen liefern wertvolle Informationen über die Umweltrelevanz von Produkten und Prozessen.

Diese Ergebnisse müssen aber verantwortlich in einen breiteren Kontext der Entscheidungsfindung eingebunden werden. Eigenes Engagement und das Einbringen der eigenen Expertise beim Auffinden von Schwachstellen und Veränderungsmöglichkeiten ist ein wesentlicher Bestandteil jeder ECODESIGN-Initiative, die durch die angebotenen Hilfsmittel unterstuitzt, aber nicht ersetzt werden kann. Umweltschutz auf Knopfdruck ist nicht möglich.

- Die am häufigsten verwendete Methodik der Bewertung und Modellierung von Umweltauswirkungen ist die Lebenszyklusanalyse (LCA).

Berücksichtigt werden vor allem Materialflüsse, Energieverbrauch und die damit verbundenen Emissionen.

Die Auswertung konzentriert sich vornehmlich auf die Beeinflussung einzelner Wirkungskategorien wie Treibhauseffekt, Ozonabbaupotential etc., oder die Berechnung aggregierter Ergebnisse, die einen Gesamtüberblick anbieten.

- Besonders gut anwendbar sind computergestützte LCA Ergebnisse für Vergleiche unter identischen Rahmenbedingungen und gleicher Fragestellung, sowie Sensitivitätsanalysen für die gezielte Untersuchung einzelner Umweltauswirkungen.

Defizite bestehen vor allem in den berücksichtigten Lebenszyklusbereichen, bei nicht quantifizierbaren Einflußparametern, der Nachvollziehbarkeit und Transparenz der Ergebnisse.

- Es sind nicht alle Lebenszyklusbereiche gleich gut abbildbar, ein deutlicher Fokus liegt auf der Herstellungsphase der Produkte.

Die Ursachen dafür sind vor allem in der verfügbaren Datenlage zu suchen, aber auch darin, daß die Rahmenbedingungen für die Herstellung wesentlich besser erfaßbar sind als etwa die der Gebrauchsphase, Distribution oder der Entsorgung. Dem Anspruch einer Bilanzierung "von der Wiege bis zur Bahre" kann nicht in voller Konsequenz entsprochen werden.

- Die Ergebnisse der computergestützten LCA Berechnungen eignen sich nicht für einen Beweis der allgemeinen Umweltverträglichkeit eines Produktes.

Dies wurde auch bei der Beispielberechnung deutlich und von einigen Software Anbietern explizit artikuliert.

- Der Umgang mit Datenunsicherheiten, Fehlern und methodischen Lücken wird erst von wenigen Programmen berücksichtigt, und zwar in Form einfacher Schwankungsbreiten.

Dem kommt besondere Bedeutung zu, da die bestehenden Unsicherheiten aufgezeigt werden und damit auch die Grenzen für die Gültigkeitsbereiche der Ergebnisse.

- Wichtig für ein aussagekräftiges Ergebnis ist eine differenzierte Fragestellung und eine möglichst detaillierte Datenaufbereitung, sowie die Einbettung der Berechnungsergebnisse in einen breiteren Kontext der Entscheidungsfindung.

\section{Zusammenfassung und Ausblick}

Die Programmqualität der untersuchten Softwarepakete kann, abgesehen von bei Beta Versionen üblichen Problemen, durchwegs als gut bezeichnet werden.

Bei den erfaßten Faktoren liegt ein deutlicher Schwerpunkt auf Materialverbrauch, Energie und Emissionen.

Entwicklungsbedarf bei den berücksichtigten Faktoren besteht insbesondere hinsichtlich der folgenden Punkte: 
- Berücksichtigung von Materialqualitäten (z. B. nachwachsende Rohstoffe)

- Flächenverbräuche

- Informationen zu Nutzungsintensität und Langlebigkeit

- Qualitative Faktoren.

Teilweise bestehen Synergieeffekte mit anderen Programmfunktionen (betriebswirtschaftliche oder technische), die den Aufwand für die ökologischen Betrachtungen reduzieren und die Akzeptanz des Programms für den betrieblichen Anwender steigern können.

Weiterer Entwicklungsbedarf für methodische Unterstützung besteht vor allem in einer stärkeren Betonung der "Produkt-Dienstleistungen", dem Nutzen, der von einem Produkt bereitgestellt wird, und in einer Entwicklung von Konzepten, die die bestehenden Instrumente einbinden in die Entwicklungsprozesse neuer Ideen und Lösungen unter Einbeziehung aller relevanten Akteure.

\section{Kontakt}

Dipl.-Ing. Robert Wimmer

GrAT Gruppe Angepaßte Technologie

TU Wien

Wiedner Hauptstr. 8-10, A-1040 Wien

Tel.: + 43-1-58801 5892

Fax: + 43-1-5869154

E-mail: r.wimmer@bigfoot.com

Die Studie kann beim Österreichischen Umweltministerium bestellt werden unter

E-mail: thomas.bertsch@bmu.gv.at

\section{Bibliographische Angaben}

Bundesministerium für Umwelt, Jugend und Familie (Hrsg.): ECODESIGN/Cleaner Production Software Recherche und Leistungstext. Endbericht. Wien 1998. ISBN 3-901 305-88-2 (Schriftenreihe des BMUJF Band 15/1998)

\section{》}


Abfälle zur Kernfrage gemacht hatte, oder sich auf die Prüfung der Umweltverträglichkeit des UTL hätte beschränken müssen. Die Genehmigungsbehörde warf Nirex vor, zu geringe Kenntnisse über die komplizierten hydrogeologischen Verhältnisse an diesem Standort aufzuweisen, verhinderte aber gleichzeitig durch die Ablehnung des UTL die Verbesserung eben dieses Kenntnisstandes. Entscheidend für die Ablehnung war schließlich noch Nirex' Konzentration auf Sellafield als einzigem Standort. Dies hatte jedoch seinen Grund darin, daß die Suche nach Standorten schon in den 80er Jahren nur in Gegenden mit kerntechnischen Anlagen wie Sellafield und Dounreay nicht am Widerstand der Bevölkerung gescheitert war. Außerdem schreiben die einschlägigen (britischen) Gesetze nicht vor, daß im Rahmen der Umweltverträglichkeitsprüfung ein Standort mit Alternativen $\mathrm{zu}$ vergleichen sei. Allerdings hatte Nirex vor 1989 mehrere Standorte an Hand einer multi-attribute decision analysis miteinander verglichen, wobei die Entscheidung zugunsten Sellafield im wesentlichen durch den Faktor Transportweg zustande kam.

In Großbritannien stellt sich nun nach der Ablehnung der Berufung die Frage, ob die geübte Genehmigungspraxis nicht die Garantie des Scheiterns beinhaltet. Der größte Teil der Insel weist kristallinen Untergrund auf, so daß es schwierig wäre zu belegen, daß grundsätzlich kein geeigneter Endlagerstandort gefunden werden könnte.

Trotzdem besteht wenig Grund zu der Annahme, daß das Genehmigungsverfahren an einem neuen Standort einen anderen Verlauf nehmen würde. Neben den verfahrenstechnischen Unwägbarkeiten verdeutlichte das Sellafield-Verfahren, daß das Hauptproblem der Endlagergenehmigung darin besteht, Konsens in der Beurteilung der, in erster Linie durch Modellrechnungen nachgewiesenen, LangzeitSicherheit herzustellen. Erwähnenswert ist an dieser Stelle die wesentliche Zielgröße - gleichzeitig auch Endlager-Auslegungskenngröße -, deren Einhaltung durch eben diese Modellrechnungen nachzuweisen ist: In Großbritannien ist diese risikobezogen definiert, es darf das individuelle Krebsrisiko zu keinem Zeitpunkt $10 \%$ Jahr überschreiten (im Vergleich dazu die deutsche Zielgröße: eine Individualdosis von $0,3 \mathrm{mSv} / \mathrm{Jahr}=30 \mathrm{mrem} / \mathrm{Jahr}$ ).
Weitgehende Übereinstimmung besteht darin, daß nur eine gründliche Neuordnung der Strukturen und Zuständigkeiten einen Ausweg bieten könnte. Kernstück müßte eine der Regierung und dem Parlament unterstellte Kommission sein, die, finanziert aber sonst unabhängig von der Nuklearindustrie, Entsorgungslösungen entwickelt und schließlich die Standortwahl trifft. Diese Kommission müßte beispielsweise auch eine Überprüfung (peer review) ihrer wissenschaftlichen Arbeit und der von ihr getroffenen Entscheidungen zulassen. "Transparenz" ist das neue Zauberwort.

Schließlich seien zwei der Gesichtspunkte erläutert, die nun in Großbritannien beim weiteren Vorgehen eine Rolle spielen werden. Der eine, die Rückholbarkeit des Abfalls aus dem Endlager, ist in den Endlagerplanungen der meisten Kernenergie-Länder vorgesehen - nicht jedoch in Deutschland, wo das möglichst schnelle Verschließen der Hohlräume durch die "Konvergenz" des Salzes ein wesentlicher Bestandteil des Konzepts ist -; der zweite, die Langzeitzwischenlagerung, gewinnt hingegen in allen diesen Ländern an Bedeutung. Beiden gemeinsam ist die Frage, wie Technik zu bewerten ist, die zu Risiken für Generationen führt, die keinen Nutzen aus dieser Technik ziehen konnten.

Bei der Rückholbarkeit geht es darum, daß einer zukünftigen Gesellschaft die Möglichkeit bewahrt werden soll, das Sicherheitsniveau zu verbessern und Material aus dem Endlager zu holen. Schwedische Behörden haben dies so formuliert, daß das Endlager ohne Überwachung und Wartung sicher sein muß, aber auch nach dem Verschließen zum Ende der Betriebsphase im Prinzip zugänglich bleiben muß; und weiter, daß die Verantwortung für das Endlager nicht diesen Generationen aufgeladen werden darf, diese jedoch "auf Wunsch" in der Lage sein müssen, Verantwortung zu übernehmen.

Langzeitzwischenlagerung wird in allen Kernenergieländern in Erwägung gezogen. In Frankreich ist sie sogar eine der drei gesetzlich vorgeschriebenen Säulen - die beiden anderen sind Tieflagerung und partitioning/transmutation -, die mit gleichem Gewicht verfolgt und 2006 dem Parlament zur Bewertung vorgelegt werden müssen. Diese Variante hilft, für die Suche nach einem Endlagerstandort Zeit zu gewinnen, durch den radioaktiven Zerfall eröff- 
net sich in manchen Länden (Frankreich, England u.a.) für einen Teil des Abfallstroms die Möglichkeit, oberflächennah (und vergleichsweise billig) endzulagern, weiter ist der Zugang zum Abfall immer möglich und schließlich werden für den Sicherheitsnachweis keine komplizierten und mit Unsicherheiten behafteten Rechenmodelle benötigt. Kritiker äußern, daß dies nicht die endgültige Lösung sein könne und somit dem Prinzip der Nachhaltigkeit klar widerspreche.

Im britischen Beispiel handelt es sich um schwachaktive Abfälle, die noch bis Mitte des nächsten Jahrhunderts ins oberflächennahe Endlager Drigg gebracht werden können, sowie um mittel- und hochaktive Abfälle - Abfälle, die tiefgelagert werden müssen -, die teils an Kernkraftwerken selbst, teils am Gelände der Nuklearanlage Sellafield zwischengelagert werden; diese Kapazitäten reichen noch 30-50 Jahre. Das Beispiel zeigt, daß keine besondere Eile bei der Standortsuche aus technischen Gründen geboten erscheint. Andererseits verstärken m.E. lange Zwischenlagerzeiten in der Öffentlichkeit den Eindruck, die Frage des endgültigen Verbleibs sei, auch technisch, noch nicht gelöst.

\section{Bibliographische Angaben}

Parliamentary Office of Science and Technology (POST): Radioactive Waste - Where Next? London 1997. 99 S. ISBN 1897941617.

\section{Die Studie kann bezogen werden über}

The Parliamentary Bookshop

12 Bridge Street, London SW1A 2JX

Tel.: + 44 (0) 71-219-3890

Fax: + 44 (0) 71-219-3866

Eine 4-seitige Zusammenfassung der Studie ist über ITAS erhältlich:

Ingrid von Berg, E-mail: berg@itas.fzk.de

Fax: + 49 (0) 7247/82-4806

\section{Studie zur globalen Entwick- lung von Wissenschaft und Technik ("Delphi '98")}

Unter diesem Titel hat Bundesminister Dr. Rüttgers im Februar dieses Jahres die vom Fraunhofer-Institut für Systemtechnik und Innovationsforschung (ISI) durchgeführte neue Delphi-Umfrage vorgestellt. An dieser zweistufigen Delphi-Umfrage haben sich in der 1. Runde 2450 Experten und in der 2. Runde 1866 Experten beteiligt. Kennzeichen einer DelphiBefragung ist, daß den Befragten, die in der 1. Befragungsrunde geantwortet haben, in einer zweiten Befragungsrunde das Meinungsbild der anderen Experten rückgekoppelt wird, so daß der einzelne Experte seine Antwort mit der Einschätzung seiner Kollegen vergleichen und ggf. überdenken kann.

Als Quintessenz der Delphi-Untersuchung hebt Bundesminister Rüttgers in seiner Pressemitteilung vom 17. Februar 1998 folgendes hervor:

- $\quad$ Die Entwicklungsdynamik in den einzelnen Bereichen ist sehr unterschiedlich. Bei Mobilität und Transport, Information, Dienstleistung und Konsum, Management und Produktion wird das kommende Jahrzehnt die größten Veränderungen bringen. Bei Energie und Rohstoffen sowie Raumfahrt fallen die erwarteten Innovationen mehrheitlich ins zweite und dritte Jahrzehnt des 21. Jahrhunderts.

- Die Experten bestätigen, daß die USA bei Wissenschaft und Technik führend sind. In bestimmten Zukunftsbereichen aber hat Deutschland die Nase vorn:

- Bei Umwelt, Energie, Bauen, Mobilität verfügt Deutschland über eine stärkere FuE-Position als die USA. Japan liegt auf Platz drei.

- Bei Chemie, Produktion, Biomedizin, Raumfahrt und Großexperimenten haben die USA zwar eine Spitzenposition, Deutschland folgt aber dicht auf Position zwei.

- Bei Information und Dienstleistungen führen ebenfalls die USA, gefolgt von Japan. Deutschland steht hier auf dem dritten Platz. 
- Nach Einschätzungen der Fachleute bleibt das Thema Beschäftigung kurz- und mittelfristig eine Achillesferse. Ohne organisatorische Veränderungen in Unternehmen, Flexibilisierung der Beschäftigungszeiten und die Wiedereinführung arbeitsintensiver Dienstleistungen sind positive Beschäftigungswirkungen nicht möglich. An der Kostenfront jedenfalls entscheidet sich das Beschäftigungsthema nicht, so die Delphi-Befragung. Dagegen wird die Bildung und ihre ständige Aktualisierung für den Einzelnen und seinen beruflichen Werdegang immer wichtiger."

Als wichtigste Ergebnisse wertet der Minister folgende:

- $\quad$ Die Unternehmensstrukturen werden sich deutlich verändern: Das Unternehmen der Zukunft wird seine Produkte, Dienstleistungen und inneren Abläufe radikal am Kunden orientieren.

Es wird - stärker als jemals zuvor - auf die Leistungsfähigkeit und Verantwortungsbereitschaft der Mitarbeiter setzen. Der Arbeitnehmer von morgen ist vielfach Mitunternehmer. Das erfordert neue innerbetriebliche Organisationsformen.

Die Beziehungen zwischen Unternehmen werden sich ändern. Stichwort sind vernetzte Unternehmen und Telearbeit. Am Standort Deutschland entstehen Produktionsverbünde mit hoher Reaktionsfähigkeit und Spezialisierung auf Kundengruppen. Insbesondere kleine und mittlere Unternehmen schließen sich zu Verbünden auf Zeit zusammen.

- Multimedia wird zur Alltagstechnik. Die technische Infrastruktur der Wissensgesellschaft wird komplett ausgebaut sein. Die Dynamik im Multimedia-Bereich wird noch größer werden. Im rund um die Uhr geöffneten elektronischen Supermarkt einzukaufen wird genauso zum Alltag gehören wie per Mausklick die Urlaubsreise zu buchen, wobei in beiden Fällen mit digitalem Geld bezahlt wird.

- Es werden neue Weiterbildungssysteme in Beruf und Alltag, also eine neue Lernkultur entstehen. Virtuelle Weltuniversitäten und Volkshochschulen sind weit verbreitet.
Systeme zum Erwerb von MultimediaInformationen "on demand" liegen weltweit in jeder der gängigsten Weltsprachen in Netzwerken dezentral bereit. Sprachübersetzungssysteme im Taschenformat machen grenzenlose Kommunikation möglich.

Die neuen Strukturen vor allem in Fortund Weiterbildung entwickeln sich mit Hilfe der Telekommunikation. Neue Möglichkeiten eröffnen sich für Menschen mittleren und höheren Alters, die mit speziellen Wiederauffrischungs- und Trainingssystemen fit bleiben können. Das "lebenslange Lernen" ist ebenso selbstverständlich wie alltäglich. Es führt vermehrt zu individuellen Qualifikationsbündeln, die mit allgemeingültigen Abschlüssen konkurrieren.

- In der zweiten Dekade des 21. Jahrhunderts sind folgende wichtige Entwicklungen zu erwarten: Für die Zeit nach 2015 zeichnen sich technische Möglichkeiten ab, ökologische Schäden zu reparieren. So wird z.B. die Wiederaufforstung der tropischen Regenwälder für möglich gehalten. Pflanzen sollen wachsen, die eine weitere Ausbreitung der Wüsten verhindern.

- Mit einem Anteil von über $10 \%$ an der Stromerzeugung werden die Erneuerbaren Energien ihren Marktanteil gegenüber heute 20fach vergrößert haben - sogar ohne Einbeziehung der Wasserkraft. Bemerkenswerte Fortschritte wird es bei der Bekämpfung ökologischer Gefahren und zur Verringerung von $\mathrm{CO}_{2}$-Emissionen geben: mit technischen Lösungen im Bereich des Fahrzeugbaus oder im Baubereich."

(Quelle: BMBF-Presse-Info v. 17.2.98)

Es kann hier nicht auf Ergebnisse im einzelnen eingegangen werden. Bundesminister Dr. Jürgen Rüttgers möchte aber Delphi zu einem zentralen Steuerungsinstrument für die mittelund langfristige Innovationspolitik machen. Deshalb sollen weitere Auswertungen des umfangreichen Datenmaterials vorgenommen werden. Zur Verbreitung der Ergebnisse sollen Workshops und Seminare beitragen, auch die Herausgabe eines Delphi-Newsletters ist geplant. Es soll eine Debatte um die Zukunftsentscheidungen in Deutschland angestoßen wer- 
den. Insgesamt werden damit hohe Erwartungen mit dem Delphi-Prozeß verbunden.

Was für die Zukunft interessant sein könnte, wäre eine systematische Analyse der Nutzung der Ergebnisse in der staatlichen und privatwirtschaftlichen Forschungs- und Technologiepolitik. Bei den vorangegangenen Delphi-Erhebungen in Deutschland hat man den Eindruck gewonnen, daß ihre Nutzung eher gering war. In anderen Ländern, z.B. Großbritannien und Schweden, werden DelphiBefragungen eher als ein ergänzendes Instrument im Vorausschauproze $\beta$ gesehen. Der Schwerpunkt wird dort auf Expertenpanel gesetzt, in denen wirkliche Kommunikation zwischen Experten stattfindet und nicht nur vermittelte.

\section{Bibliographische Angaben}

Fraunhofer-Institut für Systemtechnik und Innovationsforschung (Hrsg.): Delphi '98: Studie zur globalen Entwicklung von Wissenschaft und Technik. Zusammenfassung der Ergebnisse. Karlsruhe 1998 Ders.: Delphi '98: Studie zur globalen Entwicklung von Wissenschaft und Technik. Methoden- und Datenband. Karlsruhe 1998.

Die Studie (beide Bände) kann für DM 55,-- (zzgl. Versand und MwSt) bezogen werden beim Fraunhofer-Institut für Systemtechnik und Innovationsforschung, Breslauer Str. 48, D-76139 Karlsruhe, Fax: +49 (0) $721 / 689152$. 